\title{
1 Virulence constrains transmission even in the absence of a genetic trade-off
}

Authors

4 Diogo P. Godinho*1, Leonor R. Rodrigues ${ }^{1}$, Sophie Lefevre ${ }^{2}$, Laurane Delteil ${ }^{2}$, André F. Mira ${ }^{1}$, 5 Inês R. Fragata ${ }^{1}$, Sara Magalhães ${ }^{1,3} \dagger$ and Alison B. Duncan ${ }^{2} \dagger$

\section{Affiliations}

8 1. cE3c: Centre for Ecology, Evolution, and Environmental Changes, Faculty of Sciences,

9 University of Lisbon, Edifício C2, 3ำ piso, 1749-016 Lisboa, Portugal

10 2. Institut des Sciences de l'Évolution, Université de Montpellier, CNRS, IRD, EPHE, CC065, 11 Place Eugène Bataillon, 34095 Montpellier Cedex 05, France

12 3. Departamento de Biologia Animal, Faculdade de Ciências da Universidade de Lisboa

$13{ }^{*}$ Correspondence to: diogoprinogodinho@gmail.com

14 †(equal last author)

\section{Abstract}

17 The virulence-transmission trade-off predicts that parasite fitness peaks at intermediate virulence.

18 However, whether this relationship is driven by the environment or genetically determined and if it

19 depends on transmission opportunities remains unclear. We tackled these issues using inbred

20 lines of the macro-parasitic spider-mite Tetranychus urticae. When transmission was not possible

21 during the infection period, we observed a hump-shaped relationship between virulence and

22 parasite fitness, as predicted by theory. This was environmentally driven, as no genetic

23 correlation between traits was detected. However, when transmission to uninfected hosts

24 occurred during the infection period, virulence was positively, environmentally and genetically

25 correlated with parasite fitness. Therefore, the virulence-transmission trade-off depends on

26 within-host dynamics and on the timing of transmission, rather than on a genetic correlation. This

27 fundamental correlation may thus be easier to manipulate than previously thought. 


\section{Introduction}

30 Virulence, the harm inflicted by parasites on their hosts, is a trait with high relevance for human,

31 animal, plant and ecosystem health. It is also an evolutionary puzzle, as by harming hosts,

32 parasites seemingly jeopardize their chances of being represented in subsequent generations,

33 that is, their fitness (Alizon et al., 2009).

The most widely accepted explanation for the existence of virulent parasites is the virulence-transmission trade-off hypothesis (Alizon et al., 2009; Anderson and May, 1982). According to this hypothesis, within-host growth is a component of parasite fitness (its reproductive growth rate, $R_{0}$ ), but this is expected to entail high levels of virulence. High virulence, in turn, may lead to premature host death, hampering transmission, thus ultimately the growth of parasite populations (Anderson and May, 1982, 1979). Therefore, parasite fitness is expected to be maximised at intermediate virulence levels (Anderson and May, 1982). Despite the centrality of this hypothesis to the understanding of host-parasite interactions, and evidence of genetic variance for parasite traits (Little et al., 2008; Louhi et al., 2013; Mackinnon and Read, 1999a), whether the trade-off is due to genetic correlations among traits or is environmentally driven remains to be addressed. Disentangling these alternative possibilities is key to identify the conditions under which parasite traits can evolve independently, which could be applied on strategies for the management of parasite virulence.

Most studies in support of the trade-off hypothesis have used parasite isolates that differ genetically, but also in their recent ecological and evolutionary history, as they have different geographic origins (de Roode et al., 2008; Doumayrou et al., 2013; Ebert, 1994; Mackinnon and Read, 2003; Mackinnon and Read, 1999b). This may lead to spurious correlations between traits. Using inbred lines derived from the same parasite population allows this issue to be overcome. Additionally, most studies address the trade-off hypothesis by measuring transmission (or a proxy thereof) at the end of the infection period (Acevedo et al., 2019; Mackinnon and Read, 2003;

54 Mackinnon and Read, 1999b), which mimics a parasite with a single transmission event.

55 However, several parasites transmit continuously during the infection period (e.g. HIV, malaria

56 (Fraser et al., 2007; Mackinnon and Read, 1999b)), and this may affect the relationship between

57 virulence and transmission (Day et al., 2011). Therefore, addressing the generality of the

58 virulence-transmission trade-off requires accounting for these different parasite life cycles.

Here, we tested whether the virulence-transmission trade-off was determined by genetic correlations and/or was environmentally driven using 15 inbred lines derived from one outbred population of the spider mite Tetranychus urticae, a plant macro-parasite (Godinho et al., 2020). 
64 is visible and quantifiable through chlorotic lesions on the leaf surface ((Mira et al., 2021); Figure

65 S1), which represents a reliable measure of virulence. Once mites become adult and mate,

66 females either remain on the plant or they disperse and infect new hosts (Bitume et al., 2013).

67 Spider mites have ambulatory and passive aerial dispersal, hence transmission can depend on

68 environmental factors such as wind (Smitley and Kennedy, 1985). They can transmit during the

69 infection period or overexploit the host plant before transmission occurs (Smitley and Kennedy,

70 1985). Transmission may, thus, be dependent on many factors such as within-host parasite

71 density and/or the availability of suitable hosts to infect (Bitume et al., 2013; De Roissart et al.,

72 2015), making this system ideal to test if such factors affect the virulence-transmission trade-off.

73 Because mites are macro-parasites, we used the number of adult daughters produced in a host

74 as a measure of parasite fitness, $R_{0}$ (Anderson et al., 1986; May and Anderson, 1979). We

75 assessed whether a relationship between virulence and $R_{0}$ was determined by genetic

76 differences among lines, and/or by the build-up of density-dependence within the host, by varying

77 initial densities of infesting mites (infection dose). Additionally, we evaluated whether this

78 relationship was affected by opportunities for transmission during the infection period in two

79 separate experiments. In the first, all parasite life-history traits were measured on a single host

80 patch and no transmission was allowed until the end of the infection period. In the second, the

81 adult female offspring of the parasite could disperse continuously during the infection period to a

82 new host patch.

83 Results

84 Genetic variation for virulence, $\mathrm{R}_{0}$ and transmission

85 If the virulence-transmission trade-off is to be driven by genetic correlations among parasite traits, 86 genetic variance for these traits must be present in a parasite population. We thus measured the 87 variance for virulence, parasite fitness and transmission among the $T$. urticae inbred lines, using 88 per capita measurements, which allows broad sense heritability, $H^{2}$, to be determined. We found 89 significant genetic variance for all traits (Fig. S2). Additionally, all traits were affected by the initial 90 density of founding females on the host patch. The exception was for per capita transmission to 91 new host patches (in the experiment with continuous transmission) (Table S1). Broad sense

92 heritability was significant for all traits measured, with levels similar among experiments (Table 93 S2).

94

95 Genetic and environmental correlations between parasite traits

96 Virulence and $R_{0}$, transmission at the end of the infection period

97 We first assessed virulence and $R_{0}$ in the absence of uninfected hosts to which parasites could 98 transmit (i.e., mimicking a parasite life cycle with transmission at the end of the infection period 
only; Fig. S3): we infected hosts (bean leaf patches) with 5, 10 or 20 females from each inbred line for 4 days, then measured damage (virulence) and the number of females produced 10 days later $\left(\mathrm{R}_{0}\right)$. In support of the trade-off hypothesis, we found a hump-shaped environmental relationship (resulting from the residual co-variance of the model only) between virulence and $\mathrm{R}_{0}$. This was shown by the model with a squared term between these traits having a lower DIC, as compared to the model with only the linear term (DIC $=3379$ and 3382 , respectively). The model with the lowest DIC included density, hence this factor affected trait correlations. This was corroborated by the analysis of trait correlations for each density separately, as we found a positive correlation at low density, no relationship at intermediate density, and a negative correlation at high density (Fig. 1a, Table S3). This suggests that the relationship between these traits is modulated by density dependence: beyond a certain level of virulence, within-host competition prevents more daughters from becoming adult, such that $R_{0}$ is maximised at intermediate levels of virulence, as predicted by the trade-off hypothesis. We observed that females at higher densities do not lay fewer eggs (results not shown), suggesting that densitydependence operates during juvenile development. Evidence for density-dependence has been found in this (De Roissart et al., 2015; Rotem and Agrawal, 2003), and other host-parasite

Virulence, $R_{0}$ and transmission to uninfected hosts, continuous transmission

118 Next, for the two highest densities (10 and 20 females), we tested whether the presence of uninfected hosts (i.e. mimicking a parasite life cycle with continuous transmission during the infection period, Fig. S3) would modify the relationship between virulence and $\mathrm{R}_{0}$. Despite no overall environmental correlation between virulence and $\mathrm{R}_{0}$, this correlation was positive at both intermediate and high densities (Table S3; Fig. 1b). This suggests that the negative effects of

123 high densities on $\mathrm{R}_{0}$ were alleviated by the possibility of moving to other hosts. Additionally, this

124 correlation was affected by the inbred line identity, indicating that it has a genetic basis. Probably,

125 in these conditions, more virulent genotypes suffer less from rapid host exploitation, because they

126 can escape to new hosts. Moreover, we found a positive correlation between $\mathrm{R}_{0}$ and transmission

127 to uninfected hosts (Table S3; Fig. 2), which is in accordance with theory (Anderson and May,

128 1982).

\section{Discussion}

131 In this study we show a hump-shaped relationship between virulence and transmission that does

132 not have a genetic basis. Indeed, $\mathrm{R}_{0}$ is maximized at intermediate levels of virulence, as

133 postulated in theoretical models (Anderson and May, 1982, 1979), but this is due to within-host

134 density dependence. Moreover, this hump-shaped relationship disappears when transmission is 
continuous during the infection period. This may explain the mixed evidence for the occurrence of a trade-off in earlier studies and reinforces the idea of including the whole parasite life-cycle in the experimental set-up (Acevedo et al., 2019; Alizon and Michalakis, 2015). Indeed, if transmission timing affects the relationship between virulence and $R_{0}$, failing to include this important step of the parasite life cycle in the experimental set-up may lead to conclusions based on incomplete evidence. Contrasting relationships between virulence and $\mathrm{R}_{0}$ reliant on transmission timings may have important consequences for ecology and evolution for parasites of the same or different species.

In parasites with transmission at the end of the infection period, the lack of a genetic correlation between virulence and $\mathrm{R}_{0}$ means that selection in one trait may not affect the other, potentially maintaining variance for both traits, as observed in this (Fig. S2) and other systems (Dutta et al., 2021; Little et al., 2008; Louhi et al., 2013; Mackinnon and Read, 1999a). This may enhance the ability to cope with variability in host populations (Dutta et al., 2021; Nørgaard et al., 2021), which is particularly relevant for generalist parasites, such as T. urticae. In the absence of a genetic link with virulence, transmission may instead vary with other factors such as host availability and variability (King and Lively, 2012; Parsche and Lattorff, 2018). Conversely, selection on virulence may depend on other epidemiologically related traits, such as co-infections or the host immune system (Alizon et al., 2009). In parasites with continuous transmission, the positive genetic correlation between virulence and $R_{0}$ suggests there should be selection for higher virulence. If this is the case, why then do we still find genetic variance for this trait? We propose two non-mutually exclusive hypotheses. First, $T$. urticae is a generalist parasite, hence optimal virulence may vary with the host species (Rioja et al., 2017). Second, transmission within the infection period relies on the occurrence of hosts to which parasites can transmit. This is not necessarily always possible, as uninfected hosts may be locally absent or they may become rapidly infested (Crossan et al., 2007; Hochberg, 1991). Although mites may be passive dispersers, they base their decision to leave a host patch on their perception of cues (volatiles) from hosts in the environment, including their infection status (Kiedrowicz et al., 2017; Pallini et al., 1997). Therefore, if there are no uninfected hosts mites may remain on their host, which will eventually result in them switching to a parasite life cycle without continuous transmission. Thus, variability in transmission timing, may contribute to the maintenance of genetic variance for virulence.

The difference in parasite life cycles analyzed here has obvious implications for the virulence-transmission trade-off that have not been shown empirically before. Still, our experimental design did not include variation in the availability and/or heterogeneity of recipient hosts. Considering these host population characteristics would further contribute to our 
170 knowledge about how differences in parasite life cycles may affect the virulence-transmission

171 trade-off and, therefore, influence disease dynamics.

172

\section{Materials and Methods}

174 Biological system

175 Tetranychus urticae is an ectoparasite of over 1000 host plant species (Rioja et al., 2017).

176 Females lay eggs on the leaf surface that hatch up to 4 days later. There are three immature

177 stages, punctuated by quiescent stages, after which the mites become adult ( $\sim 9$ days from

178 hatching to adulthood in our laboratory), with their complete life cycle occurring on their host plant

179 (Helle and Sabelis, 1985). They feed by injecting their stylet into plant cells, mostly parenchyma

180 cells, and sucking out the cytoplasm, producing chlorotic lesions in the form of white spots ((Mira

181 et al., 2021), Figure S1). The damage inflicted by T. urticae (our measure of virulence), together

182 with high intrinsic growth rates, has important consequences for plant growth and yield, resulting

183 in major economic losses worldwide (Helle and Sabelis, 1985).

\section{Populations used}

Tetranychus urticae were collected on different host plants, in Portugal in 2013 (Zélé et al., 2018), and have since been reared on bean plants (Phaseolus vulgaris, variety Prelude), at the University of Lisbon. In October 2015, 50 individuals from 6 different field populations (total: 300) were collected and mixed to form an outbred population that has been kept at high densities (>1000). In October 2016, inbred lines were created from this population by sib mating. This procedure was repeated for 14 generations, ensuring an inbreeding coefficient above $94 \%$ (Godinho et al., 2020). Inbred lines allow simultaneous measurement of many individuals of the same (nearly) homozygous genotype, thus increasing the accuracy of genetic estimates (Godinho et al., 2020). Because they were derived from the same population, all lines share the same evolutionary and environmental history. Additionally, given that this population was outbred, genetic variation across lines is expected to be high (Godinho et al., 2020). Lines were maintained separately on bean leaf patches in Petri dishes. A subset of 15 inbred lines were transferred to the University of Montpellier in January 2018 and maintained on bean leaves (variety Pongo) in small plastic boxes (255 mm length $\times 183 \mathrm{~mm}$ width $\times 77 \mathrm{~mm}$ height) at optimal conditions $\left(25^{\circ} \mathrm{C}\right.$ with a $16: 8 \mathrm{~L}$ : $\mathrm{D}$ cycle, at $60 \%$ relative humidity). These same conditions were kept throughout all experiments.

Prior to the experiments, cohorts of spider mites from each inbred line were created by isolating 40 to 50 mated females of each line on bean leaves placed on water-saturated cotton wool in boxes. These females laid eggs for $48 \mathrm{~h}$. Fourteen days later, mated females (daughters) 
205

206

207

208

209

210

211

212

213

214

215

216

217

218

219

220

221

222

223

224

225

226

227

228

229

230

231

232

233

234

235

236

237

238

239

240

241

were used in the experiments. Not all inbred lines are represented in each experiment due to too few individuals available at the start of the experiment ( $\mathrm{N}$ between 12 to 14 lines).

\section{Virulence and $\mathrm{R}_{0}$, transmission at the end of the infection period}

Females of each inbred line were randomly assigned to a low, intermediate or high-density treatment, corresponding to 5, 10 or 20 founding females, respectively, on a $4 \mathrm{~cm}^{2}$ bean leaf patch, placed on wet cotton wool in plastic boxes. All females were allowed to feed and lay eggs on their leaf patches for 4 days. After this period, adult females were killed, and a photograph of each patch was taken using a Canon EOS 70D camera. The amount of damage inflicted by spider mites was measured using ImageJ (Schneider et al., 2012) and llastik 1.3 (Sommer et al., 2011). Briefly, the background from each photo was removed in ImageJ, then we distinguished damaged area from healthy leaf using llastik and finally the damaged area was calculated using the colour contrast between damaged and undamaged leaf tissue in ImageJ (Fig. S1, (Mira et al., 2021)). Control leaf patches, never exposed to spider mites, were placed in the experimental boxes for the same time period and photographed. These were used to establish an average baseline "damage", which was subtracted from each measurement, to provide an estimate of virulence. After a period of 14 days, the adult daughters surviving on each patch were counted. In this set-up, transmission would only be possible after this measurement, i.e., at the end of the infection period. There were 3 to 13 replicates for each inbred line per density, distributed across 3 blocks.

Virulence, $\mathrm{R}_{0}$ and transmission to uninfected hosts, continuous transmission

Adult females were randomly assigned to the intermediate or the high-density treatments (10 or 20 females, respectively, on a $4 \mathrm{~cm}^{2}$ bean leaf patch placed on water saturated cotton wool). As in the previous experiment, females were left to lay eggs for 4 days, after which they were killed, and a photograph was taken of each leaf to measure the damage inflicted (Fig. S1). On day 4, a second leaf patch, uninfected by spider-mites, was placed beside the first and connected to it by a $3 \times 1 \mathrm{~cm}$ Parafilm bridge (Fig. S3). In this way, the emerging adult female offspring could walk across this bridge and infect a new leaf patch. The number of adult daughters on the new host patches was checked on days 11, 12 and 13 (in block 1 only on days 12 and 13). When there were more than 15 offspring on the new patch, the latter was replaced by a new one. Host patches were replaced so that uninfected patches were always available. On day 14 , we counted the number of adult daughters on the original host patch and on each of the new patches.

Transmission was inferred by the cumulative number of females that infected a new host patch. This set-up mimics the life cycle of a parasite with continuous transmission during the infection period, as found in several systems (Fraser et al., 2007; Mackinnon and Read, 1999b). There were 5 to 16 replicates for each inbred line per density treatment, distributed across 4 blocks. 
243 Statistical analysis

244 We present correlations between T. urticae life-history traits: damage inflicted (a measure of

245 virulence), adult daughters produced (a measure of $\mathrm{R}_{0}$ for macro-parasites (Anderson et al.,

246 1986; Anderson and May, 1979)) and the number of females infecting a new host (a measure of

247 transmission). We consider total values per host patch (Table S3), which are generally used in

248 theoretical models (Anderson et al., 1986; Anderson and May, 1982; Day et al., 2011; May and

249 Anderson, 1979) and correspond to the traits measured in most experimental studies testing

250 virulence-transmission correlations (de Roode et al., 2008; Doumayrou et al., 2013; Mackinnon

251 and Read, 2003, 1999b). Genetic and environmental correlations between virulence and $R_{0}$, and

252 between $\mathrm{R}_{0}$ and transmission in the experimental set-up mimicking continuous transmission,

253 were performed using a multi-response generalized linear mixed model fitted with an MCMCglmm

254 (package MCMCglmm (Hadfield, 2010)). Genetic correlations were determined by including the

255 identity of the line as a random factor in each model and assessing the highest posterior density

256 interval (HPDI) of the genetic (G) structure of the model, which represents the (co)variances

257 between the two traits evaluated across inbred lines (Hadfield, 2010). Environmental correlations

258 were obtained by assessing the HPDI of the residual $(\mathrm{R})$ structure in the same model (Hadfield,

259 2010). Effects were considered significant when the HPDI did not overlap with 0 . The effect of

260 density on each correlation was assessed by comparing the deviance information criterion (DIC)

261 of the models including density as a fixed factor or not. We also report the genetic and

262 environmental correlations when considering each density level separately (Table S3). In

263 addition, we tested whether a non-linear regression might best describe the environmental

264 relationship between virulence and $R_{0}$ in the experiment mimicking transmission at the end of the

265 infection period only. To this aim, we compared two MCMCglmm models both with $\mathrm{R}_{0}$ fitted as the

266 response variable and inbred line and block as random factors. Both models included density as

267 a fixed factor and virulence as a covariate, with one model including only the linear term for

268 virulence and the other both the linear and the quadratic terms.

269 For the assessment of genetic variance (variance among inbred lines) and the effect of

270 density on this variance we used per capita values, by dividing the value for each host patch by

271 the initial density of females, as these values are more representative of individual variation (Fig.

272 S2, Table S2). We then applied generalized linear mixed models fitted with a Markov Monte Carlo

273 Chain approach (Hadfield, 2010). Broad-sense heritability, $H^{2}=\frac{\operatorname{Var}(G)}{\operatorname{Var}(G)+\operatorname{Var}(E)}$ (Falconer and

274 Mackay, 1996) and the corresponding confidence intervals were extracted from the

275 abovementioned models for each trait.

276 All models initially included 300000 iterations, with a burn-in of 10000 iterations, thinning of

277100 and a flat prior: For GLMMs (to assess the genetic variance within a trait), V=1 and 
$\mathrm{nu}=0.002$; For Multi-response GLMMs (to assess trait correlations), $\mathrm{V}=$ matrix $(\mathrm{c}(1,0,0,1), \mathrm{ncol}=2$, nrow $=2$ ) and nu= 0.002 . Flat priors were used to allow the hyper-parameter values to reflect a reasonable range of values for the traits in question, without any previous information about them or their co-variance. All models were checked for convergence with a stationary test using the heidel.diag function and for autocorrelations for the Markov chain within fixed and random terms using the autocorr.diag function. When models failed one of these tests, the number of iterations was increased to 500000 or to 700000 and the burn-in to 20000 or 50000 . All figures were produced with the ggplot2 package in $\mathrm{R}$ and the regressions included were fitted with the geom_smooth function (Wickham and Winston, 2016).

\section{Acknowledgments}

We deeply thank Élio Sucena, Flore Zélé, Margarida Matos, Oliver Kaltz, Sylvain Gandon and Yannis Michalakis, for their very useful suggestions and comments.

Funding

ERC (European Research Council) consolidator grant COMPCON, GA 725419 attributed to SM, PHC-PESSOA grant (38014YC) to ABD and SM, and FCT (Fundação para Ciência e Técnologia) $\mathrm{PhD}$ scholarship PD/BD/114010/2015 to DPG; This is ISEM contribution no XXXX.

\section{Author contributions}

Conceptualization and supervision by SM and ABD; Investigation by DPG, ABD, LRR, SL, AFM and LD; Formal analysis by DPG and IRF; Visualization by DG, LRR and IRF; Writing the original draft by DPG, SM and ABD with reviewing and editing by LRR and IRF.

301 Authors declare no competing interests.

\section{Data and materials availability}

303 Data will be deposited in Figshare upon acceptance.

\section{References}

Acevedo MA, Dillemuth FP, Flick AJ, Faldyn MJ, Elderd BD. 2019. Virulence-driven trade-offs in disease transmission: A meta-analysis ${ }^{*}$. Evolution (N Y) 73:636-647. doi:10.1111/evo.13692

Alizon S, Hurford A, Mideo N, Van Baalen M. 2009. Virulence evolution and the trade-off hypothesis: History, current state of affairs and the future. J Evol Biol 22:245-259. doi:10.1111/j.1420-9101.2008.01658.x

Alizon S, Michalakis Y. 2015. Adaptive virulence evolution: The good old fitness-based approach. Trends Ecol Evol 30:248-254. doi:10.1016/j.tree.2015.02.009 doi:10.1017/S0031182000055360 
Anderson RM, May RM. 1979. Population biology of infectious diseases: Part I. Nature 280:361367.

Anderson RM, May RM, Joysey K, Mollison D, Conway GR, Cartwell R, Thompson H V., Dixon B. 1986. The invasion, persistence and spread of infectious diseases within animal and plant communities. Philos Trans R Soc Lond B Biol Sci 314:533-570. doi:10.1098/rstb.1986.0072

Bitume E V., Bonte D, Ronce O, Bach F, Flaven E, Olivieri I, Nieberding CM. 2013. Density and genetic relatedness increase dispersal distance in a subsocial organism. Ecol Lett 16:430437. doi:10.1111/ele.12057

Crossan J, Paterson S, Fenton A. 2007. Host availability and the evolution of parasite life-history strategies. Evolution (N Y) 61:675-684. doi:10.1111/j.1558-5646.2007.00057.x

Day T, Alizon S, Mideo N. 2011. Bridging scales in the evolution of infectious disease life histories: Theory. Evolution (N Y) 65:3448-3461. doi:10.1111/j.1558-5646.2011.01394.x

De Roissart A, Wang S, Bonte D. 2015. Spatial and spatiotemporal variation in metapopulation structure affects population dynamics in a passively dispersing arthropod. J Anim Ecol 84:1565-1574. doi:10.1111/1365-2656.12400

de Roode JC, Yates AJ, Altizer S. 2008. Virulence-transmission trade-offs and population divergence in virulence in a naturally occurring butterfly parasite. Proc Natl Acad Sci U S A 105:7489-7494. doi:10.1073/pnas.0710909105

Doumayrou J, Avellan A, Froissart R, Michalakis Y. 2013. An experimental test of the transmission-virulence trade-off hypothesis in a plant virus. Evolution (N Y) 67:477-486. doi:10.1111/j.1558-5646.2012.01780.x

Dutta A, Croll D, Mcdonald BA, Barrett LG. 2021. Maintenance of variation in virulence and reproduction in populations of an agricultural plant pathogen. Evol App/ 14:335-347. doi:10.1111/eva.13117

Ebert D. 1994. Virulence and local adaptation of a horizontally transmitted parasite. Science (80) 265:1084-1086.

Ebert D, Zschokke-Rohringer CD, Carius HJ. 2000. Dose effects and density-dependent regulation of two microparasites of Daphnia magna. Oecologia 122:200-209. doi:10.1007/PL00008847

Falconer DS, Mackay TFC. 1996. Introduction to Quantitative Genetics. Harlow: Longman.

Fineblum W, Rausher M. 1995. Tradeoff between resistance and tolerance to herbivore damage in a morning glory. Nature 377:517-520.

Fraser C, Hollingsworth TD, Chapman R, De Wolf F, Hanage WP. 2007. Variation in HIV-1 setpoint viral load: Epidemiological analysis and an evolutionary hypothesis. Proc Natl Acad Sci U S A 104:17441-17446. doi:10.1073/pnas.0708559104

Godinho DP, Cruz MA, Charlery de la Masselière M, Teodoro-Paulo J, Eira C, Fragata I, Rodrigues LR, Zélé F, Magalhães S. 2020. Creating outbred and inbred populations in haplodiploids to measure adaptive responses in the laboratory. Ecol Evol 10:7291-7305. doi:10.1002/ece3.6454

Hadfield JD. 2010. MCMC methods for multi-response generalized linear mixed models: The MCMCglmm R package. J Stat Softw 33:1-21. 
Helle W, Sabelis MW. 1985. Spider mites: Their biology, natural enemies and control, Control. Amesterdam: Elsevier.

Hochberg ME. 1991. Non-linear transmission rates and the dynamics of infectious disease. $J$ Theor Biol 153:301-321.

Kiedrowicz A, Kuczyński L, Lewandowski M, Proctor H, Skoracka A. 2017. Behavioural responses to potential dispersal cues in two economically important species of cerealfeeding eriophyid mites. Sci Rep 7:1-9. doi:10.1038/s41598-017-04372-7

King KC, Lively CM. 2012. Does genetic diversity limit disease spread in natural host populations? Heredity (Edinb) 109:199-203.

Little TJ, Chadwick W, Watt K. 2008. Parasite variation and the evolution of virulence in a Daphnia- microparasite system. Parasitology 135:303-308. doi:10.1017/S0031182007003939

Louhi KR, Karvonen A, Rellstab C, Jokela J. 2013. Genotypic and phenotypic variation in transmission traits of a complex life cycle parasite. Ecol Evo/ 3:2116-2127. doi:10.1002/ece3.621

Mackinnon M, Read A. 2003. The effects of host immunity on virulence-transmissibility relationships in the rodent malaria parasite Plasmodium chabaudi. Parasitology 126:103112. doi:10.1017/S003118200200272X

Mackinnon M, Read A. 1999a. Selection for high and low virulence in the malaria parasite Plasmodium chabaudi. Proc R Soc B Biol Sci 266:741-748. doi:10.1098/rspb.1999.0699

Mackinnon M, Read A. 1999b. Genetic relationships between parasite virulence and transmission in the rodent malaria Plasmodium chabaudi. Evolution ( $N$ Y) 53:689. doi:10.2307/2640710

May RM, Anderson RM. 1979. Population biology of infectious diseases: Part II. Nature 280:455461. doi:10.1038/280455a0

Mira AF, Marques LM, Magalhães S, Rodrigues LR. 2021. A method to measure the damage caused by cell-sucking herbivores. Methods Mol Biol in press.

Nørgaard LS, Zilio G, Saade C, Gougat-Barbera C, Hall MD, Fronhofer EA, Kaltz O. 2021. An evolutionary trade-off between parasite virulence and dispersal at experimental invasion fronts. Ecol Lett 24:739-750. doi:10.1111/ele.13692

Pallini A, Janssen A, Sabelis MW. 1997. Odour-mediated responses of phytophagous mites to conspecific and heterospecific competitors. Oecologia 110:179-185. doi:10.1007/s004420050147

Parsche S, Lattorff HMG. 2018. The relative contributions of host density and genetic diversity on prevalence of a multi-host parasite in bumblebees. J Linn Soc 125:900-910.

Pollitt LC, Churcher TS, Dawes EJ, Khan SM, Sajid M, Basáñez MG, Colegrave N, Reece SE. 2013. Costs of crowding for the transmission of malaria parasites. Evol App/ 6:617-629. doi:10.1111/eva.12048

Rioja C, Zhurov V, Bruinsma K, Grbic M, Grbic V. 2017. Plant-herbivore interactions: A case of an extreme generalist, the two-spotted spider mite Tetranychus urticae. Mol Plant-Microbe Interact 30:935-945. doi:10.1094/MPMI-07-17-0168-CR

Rotem KA, Agrawal AA. 2003. Density dependent population growth of the two-spotted spider 
mite, Tetranychus urticae, on the host plant Leonurus cardiaca. Oikos 103:559-565. doi:10.1034/j.1600-0706.2003.12531.x

Schneider CA, Rasband WS, Eliceiri KW. 2012. ImageJ. Nat Methods 9:671-675.

Smitley DR, Kennedy GG. 1985. Photo-oriented aerial-dispersal behavior of Tetranychus urticae (Acari: Tetranychidae) enhances escape from the leaf surface. Ann Entomol Soc Am

Sommer C, Straehle C, Kothe U, Hamprecht FA. 2011. Ilastik: Interactive learning and 78:609-614. doi:10.1093/aesa/78.5.609 segmentation toolkit. Proc - Int Symp Biomed Imaging 230-233. doi:10.1109/ISBI.2011.5872394

Wickham H, Winston C. 2016. Ggplot2: Elegant graphics for data analysis., 2nd ed. Cham:

Zélé F, Santos I, Olivieri I, Weill M, Duron O, Magalhães S. 2018. Endosymbiont diversity and prevalence in herbivorous spider mite populations in South-Western Europe. FEMS 
415 Figure 1.

Density Low Intermediate $\bullet$ High

a)

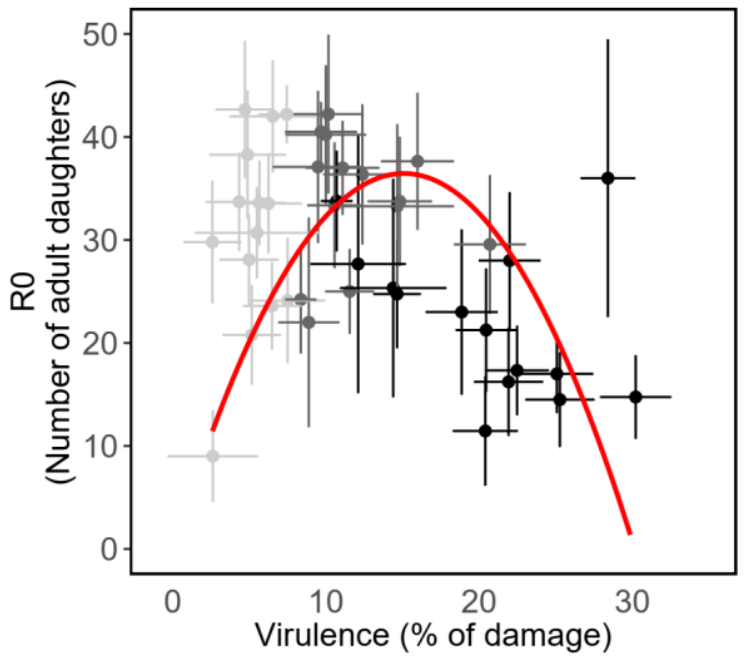

b)

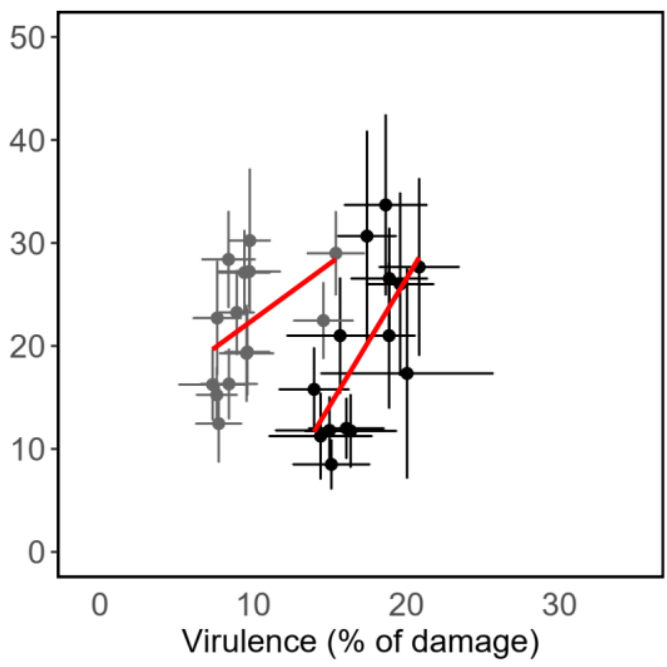

417 Figure 1. Correlation between virulence and the production of adult daughters in inbred
418 lines of $\boldsymbol{T}$. urticae infecting a host patch. The damage inflicted on the host patch (i.e.,

419 virulence) and the number of daughters produced (the parasite reproductive rate $R_{0}$ ) were

420 measured in a set-up with a) no uninfected hosts available; b) uninfected hosts available during

421 the infection period. Shades of grey represent different densities; dots are the mean for each

422 inbred line \pm standard error; regressions are represented in red. 
$424 \quad$ Figure 2.

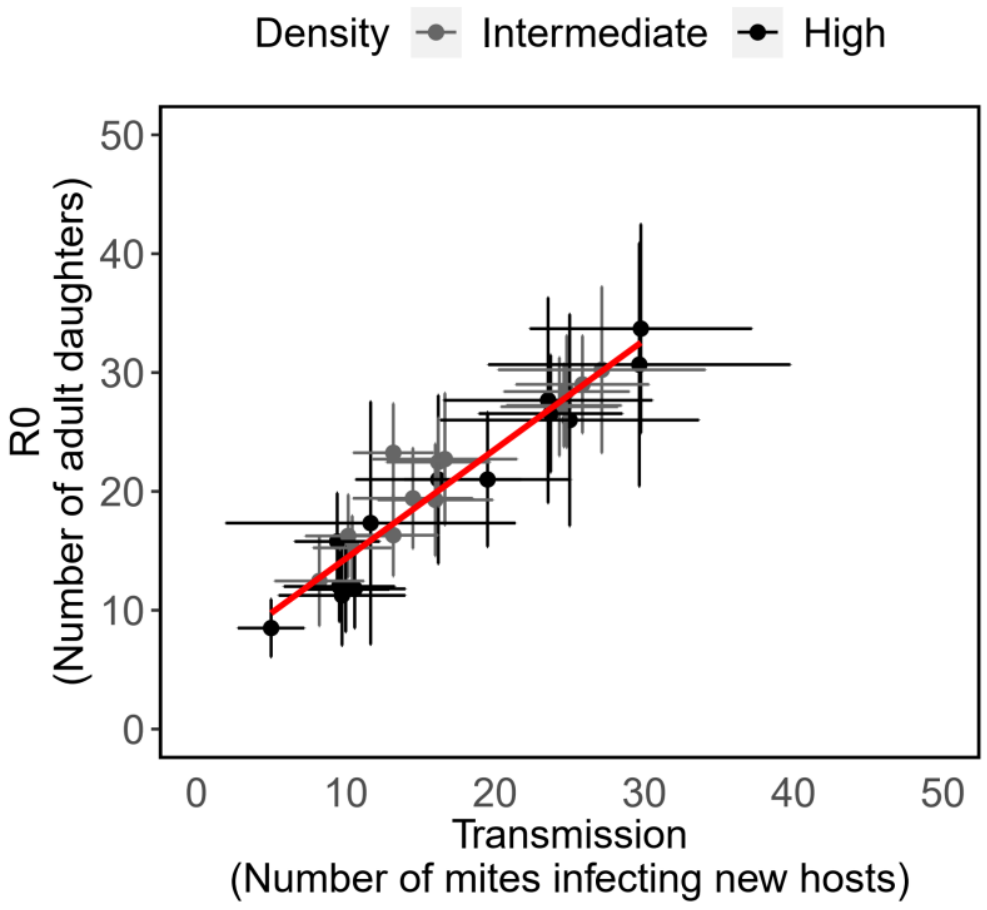

Figure 2. Correlation between the production of adult daughters and transmission in

427 inbred lines of $\boldsymbol{T}$. urticae infecting a host patch. The number of daughters produced (the

428 parasite reproductive rate, $R_{0}$ ) and transmission (the number of mites infecting new hosts) for

429 inbred lines of $T$. urticae at different starting densities (Intermediate $=10$ females; High $=20$

430 females). Shades of grey represent different densities; dots are the mean for each inbred line \pm

431 standard error; the regression is represented in red. 


\section{Supplementary Information for}

\section{"Virulence constrains transmission even in the absence of a genetic trade-off"}

\section{Authors}

Diogo P. Godinho*1, Leonor R. Rodrigues ${ }^{1}$, Sophie Lefevre ${ }^{2}$, Laurane Delteil ${ }^{2}$, André F. Mira ${ }^{1}$, Inês R. Fragata ${ }^{1}$, Sara Magalhães ${ }^{1,3} \dagger$ and Alison B. Duncan ${ }^{2} \dagger$

\section{Affiliations}

1. cE3c: Centre for Ecology, Evolution, and Environmental Changes, Faculty of Sciences, University of Lisbon, Edifício C2, $3^{\circ}$ piso, 1749-016 Lisboa, Portugal

2. Institut des Sciences de l'Évolution, Université de Montpellier, CNRS, IRD, EPHE, CC065, Place Eugène Bataillon, 34095 Montpellier Cedex 05, France

3. Departamento de Biologia Animal, Faculdade de Ciências da Universidade de Lisboa

*Correspondence to: diogoprinogodinho@gmail.com

†(equal last author)

\section{This file includes:}

Figures $\mathrm{S} 1$ to $\mathrm{S} 3$

Tables S1 to S3 

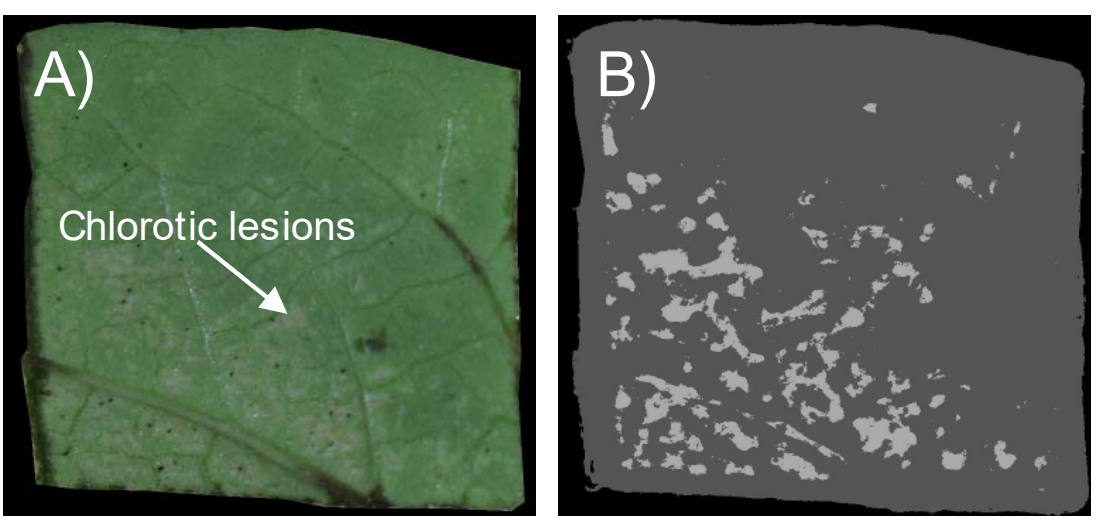

3 Figure S1. Example of a host patch cut from a bean plant (Phaseolus vulgaris) upon

4 image acquisition and after software output. A) Leaf damage (chlorotic lesions)

5 caused by T. urticae feeding. B) The photograph is transformed into a simple

6 segmentation image using Ilastik 1.3. Areas of leaf are damage shown in light grey and

7 correspond to our measure of virulence. 

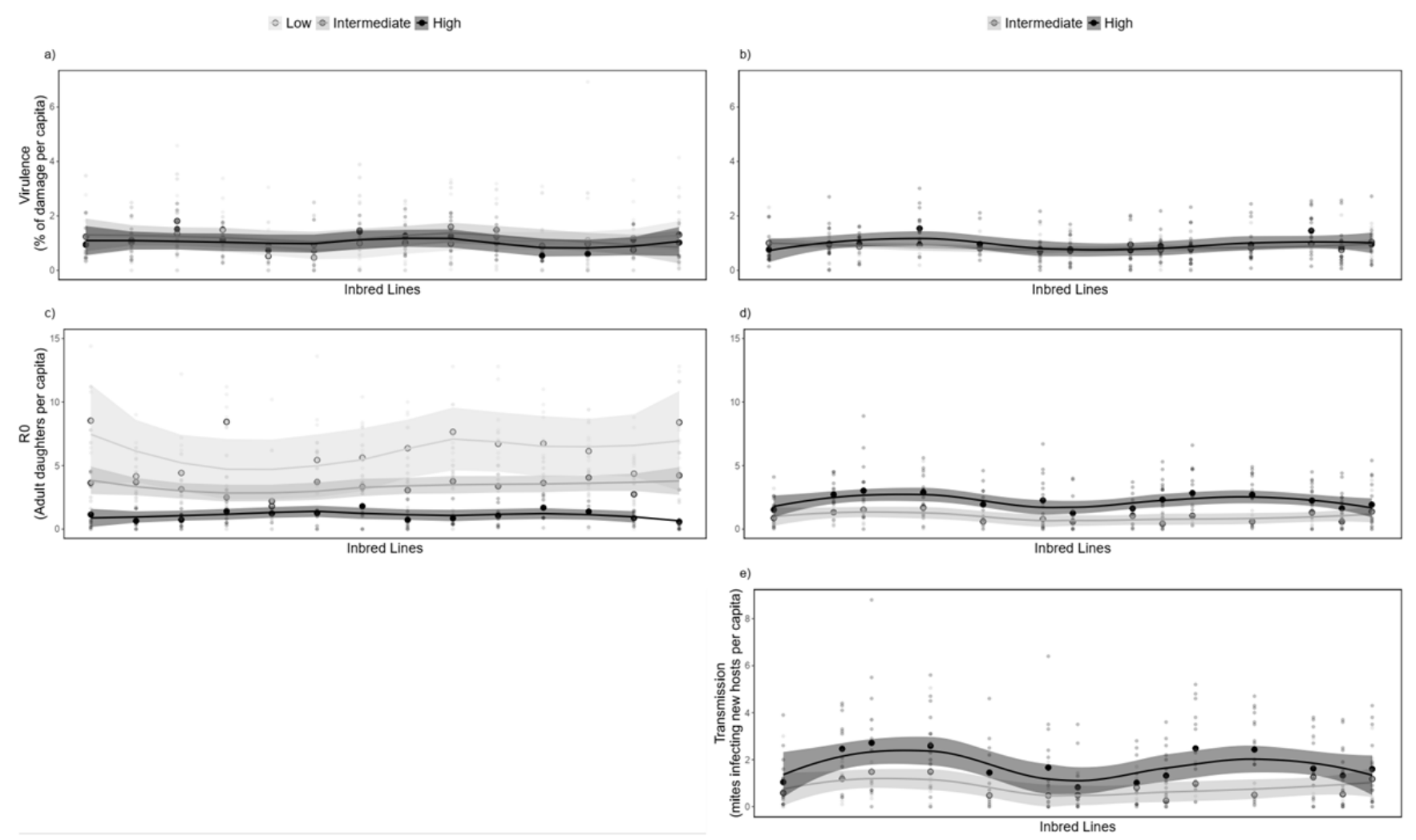

9 Figure S2. Genetic variance. Genetic variance for virulence ( $\%$ of damage inflicted; a and b), $\mathrm{R}_{0}$ (the number of adult daughters; $\mathrm{c}$

10 and d), and transmission (number of mites infecting new hosts; e), measured per capita in the different experiments (Experiment 1 - a

11 and c; Experiment $2-b, d$, and e). Large circles represent the average per line, per density (light grey $=$ Low density; dark grey $=$

12 Intermediate density; black = High density). The shape of the curve for each density (light grey = Low density; dark grey =

13 Intermediate density; black = High density) was calculated using a polynomial regression fitted with the geom_smooth function. 
1. Virulence and $R_{0}$, transmission at the end of the infection period

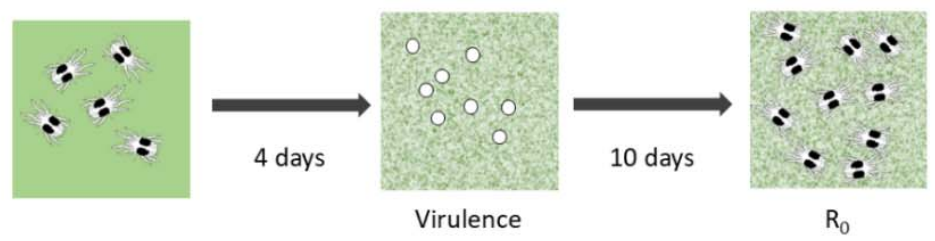

2. Virulence, $R_{0}$ and transmission to uninfected hosts, continuous transmission

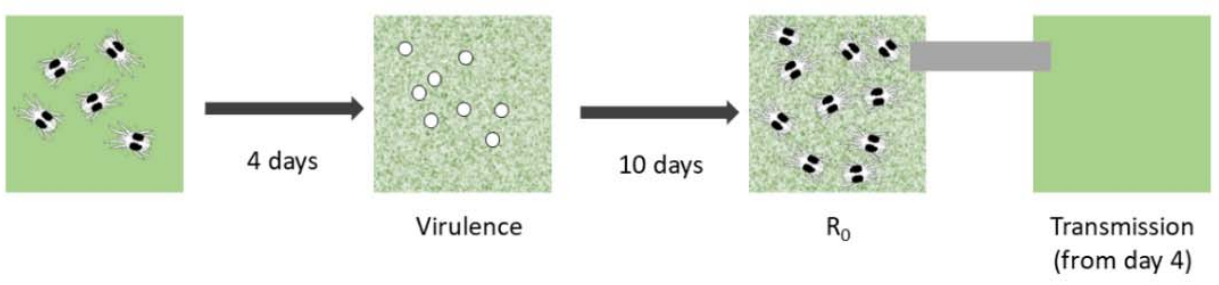

15 Figure S3. Experimental set-ups. Schematic representation of the experimental set-ups

16 and the traits that were measured in each experiment. Day 0 shows 5 spider mites on a

17 healthy $4 \mathrm{~cm}^{2}$ leaf patch (low density treatment). On day 4 , virulence (mottled white areas

18 on leaf patches) was measured in experiments 1 and 2 . $\mathrm{R}_{0}$ (i.e. number of adult daughters)

19 was measured 14 days after mite installation. In experiment 2 transmission to an

20 uninfected leaf patch was possible from day 4 to day 14 . 
22 Table S1. The effect of the initial density of female spider mites on the patch on the 23 per capita production of adult daughters $\left(\mathrm{R}_{0}\right)$, virulence and transmission (i.e. "trait" in 24 the model column). Deviation information criterion (DIC) of the models (MCMCglmm package) measured in the different experiments with density included or not as a fixed

26 factor. When applicable, best fit models are in bold. Experiment 1: transmission at the

27 end of the infection period only; experiment 2: continuous transmission.

\begin{tabular}{|c|c|c|c|}
\hline Trait & Experiment & Model & DIC \\
\hline \multirow{4}{*}{ Adult daughters } & \multirow[b]{2}{*}{1} & trait $\sim-1+$ random $=$ Line + Block & 2070 \\
\hline & & $\begin{array}{l}\text { trait } \sim-1+\text { Density }+ \text { random }=\text { Line }+ \\
\text { Block }\end{array}$ & 1835 \\
\hline & \multirow[b]{2}{*}{2} & trait $\sim-1+$ random $=$ Line + Block & 1217 \\
\hline & & $\begin{array}{l}\text { trait } \sim-1+\text { Density }+ \text { random }=\text { Line }+ \\
\text { Block }\end{array}$ & 1147 \\
\hline \multirow{4}{*}{ Virulence } & \multirow[b]{2}{*}{1} & trait $\sim-1+$ random $=$ Line + Block & 3019 \\
\hline & & $\begin{array}{l}\text { trait } \sim-1+\text { Density }+ \text { random }=\text { Line }+ \\
\text { Block }\end{array}$ & 2824 \\
\hline & \multirow[b]{2}{*}{2} & trait $\sim-1+$ random $=$ Line + Block & 464 \\
\hline & & $\begin{array}{l}\text { trait } \sim-1+\text { Density }+ \text { random }=\text { Line }+ \\
\text { Block }\end{array}$ & 456 \\
\hline \multirow[b]{2}{*}{ Transmission } & \multirow[b]{2}{*}{2} & trait $\sim-1+$ random $=$ Line + Block & 2762 \\
\hline & & $\begin{array}{l}\text { trait } \sim-1+\text { Density }+ \text { random }=\text { Line }+ \\
\text { Block }\end{array}$ & 2763 \\
\hline
\end{tabular}


30 Table S2. Trait heritability. Broad-sense heritability for the per capita production of

31 adult daughters $\left(\mathrm{R}_{0}\right)$, virulence and transmission measured in the different experiments.

$3295 \%$ highest posterior density intervals (HPDI) intervals for the heritability of each trait.

33 All traits have significant heritability as no interval includes zero. Experiment 1:

34 transmission at the end of the infection period only; experiment 2: continuous

35 transmission.

\begin{tabular}{|c|c|c|c|}
\hline Trait & Experiment & Heritability & 95\% HPDI \\
\hline \multirow{2}{*}{ Adult daughters } & 1 & 0.09 & $0.001,0.19$ \\
\cline { 2 - 4 } & 2 & 0.07 & $0.001,0.15$ \\
\hline \multirow{2}{*}{ Virulence } & 1 & 0.03 & $0.001,0.07$ \\
\cline { 2 - 4 } & 2 & 0.06 & $0.001,0.12$ \\
\hline Transmission & 2 & 0.14 & $0.02,0.27$ \\
\hline
\end{tabular}

36 
Table S3. Genetic and environmental correlations. Genetic and environmental correlations - extracted from the genetic (random) or residual error structure of the models, respectively - between the production of adult daughters $\left(\mathrm{R}_{0}\right)$ and virulence or transmission. All traits were measured per host (total value). The deviance information criterion (DIC) of models for all data with and without densities are shown. Highest posterior density intervals (HPDI) are shown for the model including all data (* model with lowest DIC, or in the case of $\mathrm{R}_{0}$ vs. transmission, the simplest model), and separately at the different densities. Intervals not overlapping 0 are shown in bold. The direction of the correlations is shown in brackets. Experiment 1: transmission at the end of the infection period only; experiment 2: continuous transmission.

\begin{tabular}{|c|c|c|c|c|c|c|c|c|c|c|c|}
\hline \multirow[b]{3}{*}{ Traits } & \multirow[b]{3}{*}{ Experiment } & \multicolumn{4}{|c|}{ All } & \multicolumn{2}{|c|}{ Low density } & \multicolumn{2}{|c|}{ Intermediate density } & \multicolumn{2}{|c|}{ High density } \\
\hline & & \multicolumn{2}{|c|}{ DIC } & \multicolumn{8}{|c|}{ HPDI } \\
\hline & & $\begin{array}{c}\text { No } \\
\text { density }\end{array}$ & Density & $\begin{array}{c}\text { genetic } \\
\text { covariance } \\
\text { (G) }\end{array}$ & $\begin{array}{l}\text { residual } \\
\text { covariance } \\
\text { (R) }\end{array}$ & $\begin{array}{c}\text { genetic } \\
\text { covariance } \\
\text { (G) }\end{array}$ & $\begin{array}{c}\text { residual } \\
\text { covariance } \\
\text { (R) }\end{array}$ & $\begin{array}{c}\text { genetic } \\
\text { covariance } \\
\text { (G) }\end{array}$ & $\begin{array}{c}\text { residual } \\
\text { covariance } \\
\text { (R) }\end{array}$ & $\begin{array}{c}\text { genetic } \\
\text { covariance } \\
\text { (G) }\end{array}$ & $\begin{array}{c}\text { residual } \\
\text { covariance } \\
\text { (R) }\end{array}$ \\
\hline \multirow{2}{*}{$\begin{array}{c}\text { Virulence vs } \\
\mathrm{R}_{0}\end{array}$} & 1 & 6297 & $6187 *$ & $-0.99,0.99$ & $-0.20,0.01$ & $-0.99,0.99$ & $\begin{array}{c}0.12,0.42 \\
(+)\end{array}$ & $-0.99,0.99$ & $-0.11,0.22$ & $-0.99,0.72$ & $\begin{array}{l}-0.38,- \\
0.005(-)\end{array}$ \\
\hline & 2 & 4936 & $4856^{*}$ & $\begin{array}{c}0.97,0.99 \\
(+)\end{array}$ & $-0.11,0.11$ & na & na & $\begin{array}{c}0.81,0.99 \\
(+)\end{array}$ & $\begin{array}{c}0.12,0.17 \\
(+)\end{array}$ & $\begin{array}{c}0.52,0.99 \\
(+)\end{array}$ & $\begin{array}{c}\text { 0.10, } 0.27 \\
(+)\end{array}$ \\
\hline$\underset{\text { transmission }}{\mathrm{R}_{0 \mathrm{vs}}}$ & 2 & 3632 & 3632 & $\begin{array}{c}0.94,0.99 \\
(+)\end{array}$ & $\begin{array}{c}0.99,0.99 \\
(+)\end{array}$ & na & na & $\begin{array}{c}0.48,0.99 \\
(+)\end{array}$ & $\begin{array}{c}0.99,0.99 \\
(+)\end{array}$ & $\begin{array}{c}0.81,0.99 \\
(+)\end{array}$ & $\begin{array}{c}0.99,0.99 \\
(+)\end{array}$ \\
\hline
\end{tabular}

